\title{
Gait Intention Analysis for Controlling Virtual Reality Walking Platforms
}

\author{
Laura Madalina Dascalu ${ }^{1}$, Adrian Stavar ${ }^{1}$, and Doru Talaba ${ }^{2}$ \\ 1,2 Transilvania University of Brasov, Product Design and Robotics Department, \\ Bulevardul Eroilor, nr. 29, 500036, Brasov, Romania \\ \{madalina.dascalu, adrian.stavar, talaba\} @unitbv.ro
}

\begin{abstract}
Simple human gait can be difficult, unfeasible and not always practical in Virtual Reality, because of spatial and technological limitations. For 3D virtual environment travelling, different walking platforms have been developed in the last few years, but navigation using most of them is far from bringing naturalness to the user's movements. Users sometimes are unsecure and they are trying to adapt and to correct any irregularities they feel. Our research is focused on specific walking patterns that characterize the intention of walking: starting walking with a certain speed, maintaining a desired speed, accelerated walking, decelerated walking, stopping. In laboratory conditions, using a motion capturing system, these behaviors were reproduced, measured and analyzed.
\end{abstract}

Keywords: human gait, Virtual Reality, walking patterns, walking platforms, walking intention, gait analysis, kinematics.

\section{Introduction}

The main motor activity of daily life, human walk, is a complex, dynamic and unforeseeable process. It involves multiple joints movements, demands sensory-motor integration and the synchronization of the skeleton with the neurological system. Even if it runs mostly unconsciously, it has important goals: to keep upright, to maintain balance, to avoid collapse, to move the center of mass forward, change direction when necessary, avoid obstacles, adapt for avoiding painful forces or motions etc [1]. Human gait is integrated in a big, autonomous and auto-adaptive control system, capable of learning, which is the human being.

The complexity and difficulty of human gait have made it difficult to use as a key element present in the natural human-machine interaction. In Virtual Reality (VR), human gait is considered the user's most natural way of exploring a virtual environment [2], [3]. The user does not have to learn any interaction metaphor [4]; he can use his own walk for navigating inside the virtual environment, in the same way as he does in the real environment.

For Virtual Reality travelling, multiple devices and platforms have been built, to facilitate the use of human natural walk as the main way of navigating and exploring a $3 \mathrm{D}$ virtual environment. Though, most existing platforms are either too large or 
expensive, or don't bring naturalness and safety to the users' movements during travelling [5], [6], [7], [8]. To create to the user the perception of natural walking in a virtual environment, a VR travelling platform should permit the user to walk freely, in any direction and with any desired speed, but this is not always possible because of physical and technical limitations.

The control system must have an appropriate response regarding the user's locomotion, in order to assist him in a freely and unrestricted manner, but at the same time to keep him/her as precise as possible in a fixed point and area of the walking surface. In this case one of challenges for the controlling system is related to the various changes of speed and direction in human natural walking. The main question is whether it is possible or not to anticipate and predict the gait intent in order to allow a real time control for high precision gait cancelling.

The attention of this paper is focused on the various changes of the linear walking speed. It is presented an approach for gait intent identification based on classification of the human postures while taking various decisions for linear walking. Analyzing the specific walking inputs that the control systems of travelling platforms use [5], [6], [7], [8] we observed that they are not focused on specific decision patterns that characterize the intention of walking: starting walking with a certain speed, maintaining a desired speed, accelerated walking, decelerated walking, stopping. These locomotion intentions can be externally observed in user's walking behaviors. In laboratory conditions, using a motion capturing system, these decision walking behaviors were reproduced, measured and analyzed.

\section{Contribution to Sustainability}

Virtual Reality is one of the most promising simulation technologies for the future sustainability. The application areas range from medical, engineering to urban planning, training and education, sports and arts. Natural navigation of the human user in Virtual Environments is one of the current bottlenecks which are expected to unlock a large number of applications on the entire spectrum but especially in the area of urban planning and built environment which are crucial for the future sustainable development.

One of the latest applications of gait study concerns the natural navigation in virtual environments using special devices like carpets and treadmills that are able to cancel the human displacement while walking. The main problem for the control of these devices is the identification of human gait intent in order to compensate in real time the displacement with the highest possible precision. Usually, the latency in anticipation of the displacement intent leads to the necessity of a device with a larger walking area. The more precise is the anticipation of the gait intent the smaller the needed walking area is needed. In case of Virtual Reality applications with limited user space, increasing the speed and precision of anticipation is crucial, as the user have a limited physical space available for walking.

The proposed approach, presented in this paper, is focused on building better controlling systems for Virtual Reality travelling platforms that will adapt to the user needs and will eliminate the user's intervention for machine accommodation. This paper presents results of preliminary tests for linear walking, which are promising for 
further development of our project: obtaining the instantaneous parameters for omnidirectional walking. Nevertheless, identifying the gait intent is a very complex issue that not only needs much more information about the instantaneous postures, but also interpretation of these data such as to be able to predict the next moves.

\section{Methodology}

\subsection{Participants}

Five healthy young subjects were recruited as volunteers from the student population of Transilvania University of Brasov, Romania. Before participating in the experiments, the subjects were fully informed about the nature of the study and they all gave their consent to participate to it. The mean age of the participants was 25 . The participants wore comfortable shoes and tight fitting clothing for reducing the movements of the markers placed on the body (Fig. 1).
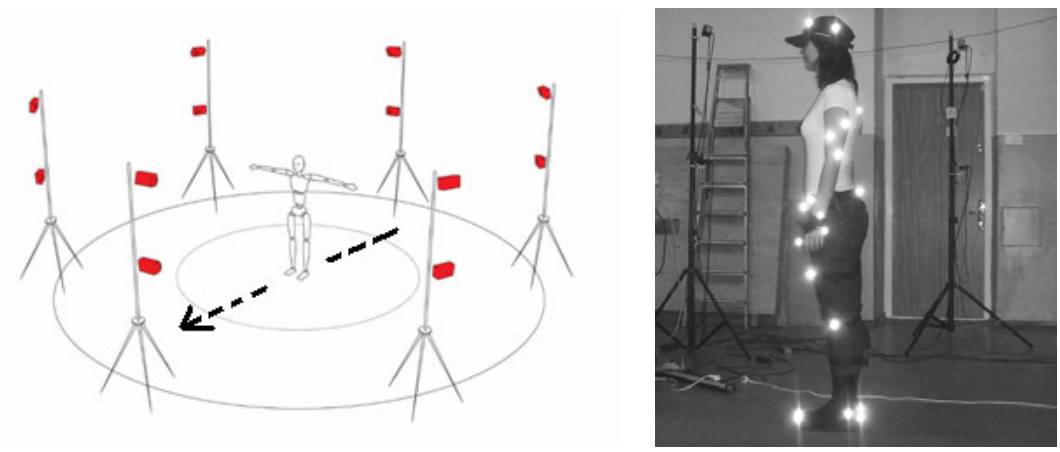

Fig. 1. Left: Camera placement (IR cameras in portrait orientation), capturing area and subject's walking direction (dotted line). Right: Full body marker placement (lighting points).

\subsection{Apparatus}

Human walking was measured using the Optitrack Motion Capturing System [9]. Optitrack Motion Capturing System is a passive optical system that use markers coated with a retro-reflective material to reflect light back to the infrared cameras (IR). Twelve IR cameras were used for data capture (as it can be seen in the Fig. 1).

The optical motion capturing system was chosen for the main advantages it has: high update rates, low latency and scalable to fairly large areas [10]. The surrounding environment was designed carefully to reduce ambient radiation, the main disadvantage of this motion capturing system.

A stationary laboratory coordinate system was defined by a vertically oriented $\mathrm{Y}$ - axis, a Z-axis placed forward in the walking direction, and an $\mathrm{X}$-axis perpendicular to the first two. A calibration volume was set to $2.50,2.50$, and $2.20 \mathrm{~m}$. The motion analysis model used was a full body human model with 34 markers set, placed on specific landmarks [9], [10] of the body (full body marker placement is shown in 
Fig. 1). For higher precision in marker capturing a skeleton was used. The skeleton tracking rate is of 100 FPS (frame per seconds) for the FLEX:V100 cameras used in the presented study.

\subsection{Test Scenario}

All testing were performed in the Virtual Reality Research Laboratory from Transilvania University of Brasov, Romania. Subjects had to perform some overground linear walking on the laboratory floor, according to specific received instructions. The instructions received by the participants refer to: starting and stopping linear walking motion at their self-selected moment of time, performing some linear walking at their desired speed and then accelerate (at their self-selected speed) or decelerate (at their selected speed), performing an accelerated linear walking and then decelerate, performing a decelerated linear walking and then accelerate.

The aim of this experimental research was to reproduce, in a controlled environment, the linear walking movements a user does when exploring an environment (it can be real or virtual). There were considered only a limited number of linear walking states, being selected only those considered relevant for VR navigation. These linear walking states with their characterization are presented in Table 1. There were also analyzed the transitions from one state to another, in the same context discussed above.

Table 1. Linear walking states

\begin{tabular}{|c|c|c|}
\hline Linear walking state & & State characterization \\
\hline Repose & $\mathrm{R}$ & $\begin{array}{l}\text { The subject is in repose state when his velocity } \\
\mathrm{v}=0 \text {. }\end{array}$ \\
\hline Constant walking & $\mathrm{CW}$ & $\begin{array}{l}\text { The subject is in constant walking state when } \\
\text { the mean value of its Center of Mass (CoM) } \\
\text { velocity is constant }\end{array}$ \\
\hline Accelerated walking & AW & $\begin{array}{l}\text { The subject is in accelerated walking state } \\
\text { when the mean value of its Center of Mass } \\
\text { velocity is increasing from one gait cycle to the } \\
\text { next one }\end{array}$ \\
\hline Decelerated walking & DW & $\begin{array}{l}\text { The subject is in decelerated walking state } \\
\text { when the mean value of its Center of Mass } \\
\text { velocity is decreasing from one gait cycle to the } \\
\text { next one }\end{array}$ \\
\hline
\end{tabular}

\section{Results and Discussion}

All experiments were conducted in controlled laboratory conditions. Specific walking scenarios were reproduced and measured using real-time motion capture data from a marker-based optical motion capture system, the Optitrack Motion Capturing System. Kinematic procedures are used for measuring the spatial motion of the full body and of its segments, during the representative linear walking states. Temporal and spatial kinematic gait parameters provide information about the time and position of the persons' gait. 
Previous studies analyzed gait initiation and gait termination in terms of balance and control [11], [12], [13] but little was done in terms of gait initiation with the aim of controlling travelling platforms for VR. Gait initiation represents the transition from the repose state (see Table 1) to the CW state, AW state or DW state, all of them, being a periodic movement from one base of support to another, the gait cycle.

A gait initiation sequence can be observed in Fig. 2. Analyzing the lower extremities of the body, the first movement in gait initiation can be observed at the knee level, but the transition movement from the repose state to first heel contact (HC) of gait cycle can be observed only at foot level, when the right foot is up and the left foot is in inverted pendulum support, supporting the body weight. The left foot initial push is moving the body's CoM forward and to the right [11]. This is forcing the right leg to get from swinging to heel contact, characterized by the heel marker position close to the ground.
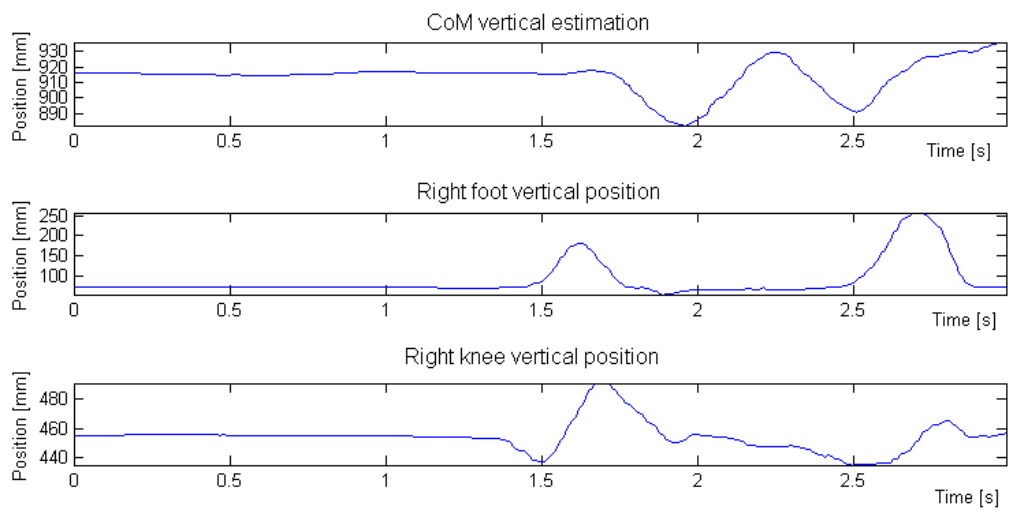

Fig. 2. Lower extremities marker displacements during gait initiation

The desired speed is achieved only after the next heel contact of the right leg. The periodicity of the same markers is confirming this statement. The autocorrelation function for the knee marker displacement shows a periodic movement, as it can be seen in Fig. 3.

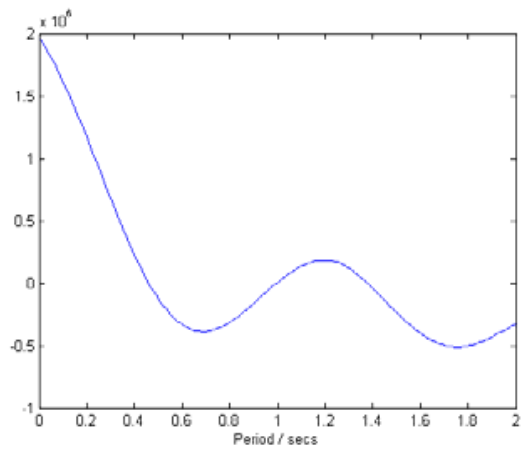

Fig. 3. Estimated periodicity of the movement of the knee marker 
Autocorrelation function for the heel and knee markers revealed no periodic movement along both the vertical and horizontal dimensions at slow walking motions, but only on trunk marker, which can be theoretically explained by the periodicity of the CoM displacement during gait cycle [1], [14], [15], [16].

Gait termination (normally on double feet support) involves on one side, stance phase from the associated leg, completed on the other side with a shortened swing phase from the opposite leg and its placing next to the first one. The stopping sequence is much difficult to measure in terms of prediction using specific kinematic gait patterns. Coming back to the repose state is achieved only through a decelerated walking state which can be observed. The effective gait termination can be on the same foot with gait initiation or on the opposite foot. Fig. 4 shows a return to the repose state from the perspective of the stance support leg and of the opposite leg.

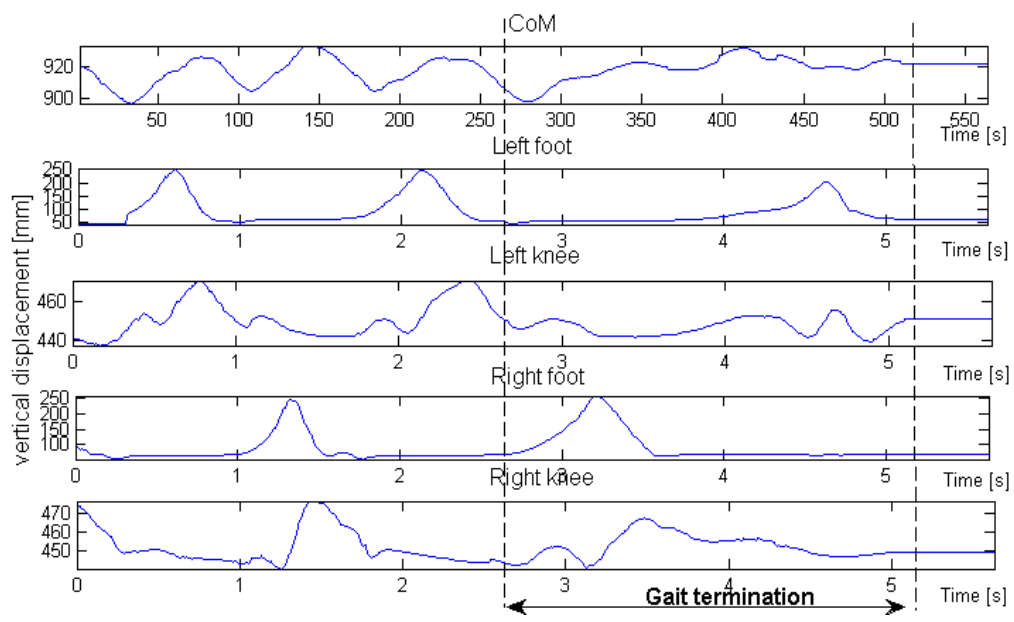

Fig. 4. Estimated periodicity of the movement of the knee marker

Gait termination presented in Fig. 4 starts with a heel contact of the left foot - the stance support leg. At this moment all the feet parameters are the same as in the periodic gait cycle, but it can be observed a smaller displacement at the level of the right knee. This leads to the idea of taking into account the position of the both feet when analyzing the transition from walking to repose state.

A left foot balance can be also observed after the heel contact of the opposite leg. This can be theoretically explained by applying a backwards and leftwards force to the body center of gravity, arresting its forward motion and bringing it to the midpoint between the feet [11]. Even it has been shown that there are asymmetries between the two sides of the body [17] for the purpose of this study these asymmetries can be neglected.

A transition sequence, from $\mathrm{CW}$ to $\mathrm{AW}$, and then to $\mathrm{DW}$ and stopping can be observed in Fig. 5 in terms of velocities. The increasing of the shank's speed is come along with an increasing of the user's speed. Also, a higher decreasing of shank's speed is necessary for decreasing the user's speed. 


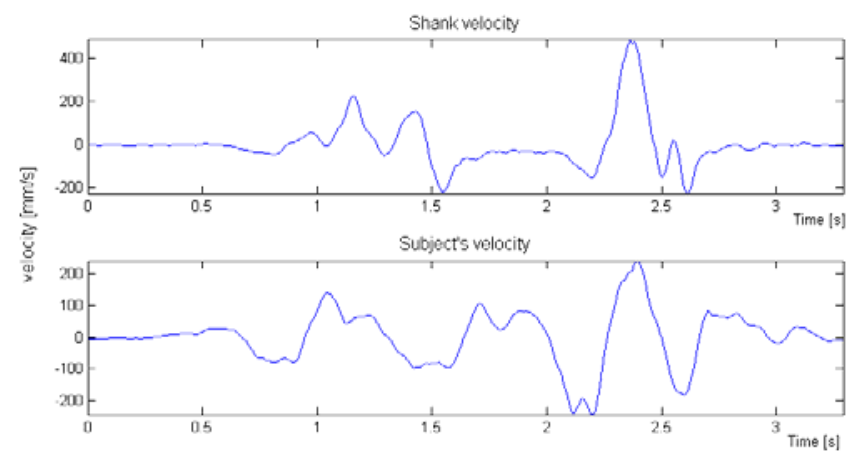

Fig. 5. Estimated periodicity of the movement of the knee marker

\section{Conclusions and Future Work}

The presented research from this paper represents the early results of an ongoing research project at Transilvania University of Brasov and brings into attention the identification and prediction of human linear walking for building better controlling systems for Virtual Reality walking platforms. The aim of the present study was to get a measure of the natural linear walking, in transitions from repose to steady-state continuous walking, or from accelerated walking to decelerated walking and then to repose again etc. There were of interest only the aspects of the human linear walking; the omni-directional walking parameters in the context discussed are subjects of our further research.

The method described in this paper and the proposed directions in identifying user intent aims to achieve to a new level of understanding human gait, dedicated to realtime prediction of gait intention and real-time control and command of VR platforms inside an immersive environment. This level is centered on observable intentions of human walking: the measured human motor actions.

Acknowledgments. This paper is supported by the Sectoral Operational Programme Human Resources Development (SOP HRD), financed from the European Social Fund and by the Romanian Government under the contract number POSDRU/6/1.5/S/6 for the authors (1) and by the research project IREAL contract no. 97/2007, id:132, funded by the Romanian Council for Research CNCSIS for the authors (2).

\section{References}

1. Vaughan, C.L., Connor, O., Davis, J.C., Dynamics, B.L.: Human Gait. PublishersSmith, Kiboho (1999)

2. Usoh, M., Arthur, K., Whitton, M.C., Bastos, R., Steed, A., Slater, M., Frederick, P., Brooks, J.: Walking > walking-in-place $>$ Flying, in virtual environments. In: Proceedings of the 26th Annual Conference on Computer Graphics and Interactive Techniques, SIGGRAPH 1999, pp. 359-364. ACM Press/Addison-Wesley Publishing Co, New York (1999) 
3. Heintz, M.: Real Walking in Virtual Learning Environments: Beyond the Advantage of Naturalness. In: Cress, U., Dimitrova, V., Specht, M. (eds.) EC-TEL 2009. LNCS, vol. 5794, pp. 584-595. Springer, Heidelberg (2009)

4. Sherman, W.R., Craig, A.B.: Understanding Virtual Reality. Elsevier Science, USA (2003)

5. De Luca, A.: The Motion Control Problem for the CyberCarpet. In: Proceeding of the 2006 IEEE International Conference on Robotics and Automation, pp. 3532-3535. IEEE Press, Florida (2006)

6. CyberWalk Project, http: / /www. cyberwalk-project.org

7. The String Walker Project, http://intron.kz.tsukuba.ac.jp/stringwalker/

8. CirculaFloor, http://intron.kz.tsukuba.ac.jp/CirculaFloor/CirculaFloor_j. htm

9. Optitrack Motion Capturing System, http: / /www. naturalpoint.com/optitrack

10. Medved, V.: Measurement of Human Locomotion. CRC Press, New York (2001)

11. Winter, D.A.: Human balance and posture control during standing and walking. Gait and Posture 3, 193-214 (1995)

12. Elble, R.J., Moody, C., Leffler, K., Sinha, R.: The Initiation of Normal Walking. Mov. Disord. 9(2), 139-146 (1994)

13. Breniere, Y.: When and How Does Steady State Gait Movement Induced from upright posture begin? Journal of Biomechanics 19(12), 1035-1040 (1986)

14. Halvorsen, K., Eriksson, M.: Minimal Marker Set for Center of Mass Estimation in Running. Gait and Posture 30, 552-555 (2009)

15. Farley, C.T., Ferris, D.P.: Biomechanics of Walking and Running: Center of Mass Movements to Muscle Action. Exercises and Sport Sciences Reviews 26, 253-285 (1998)

16. Forsell, C., Halvorsen, K.: A Method for Determining Minimal Sets of Markers for the Estimation of Center of Mass, Linear and Angular Momentum. Journal of Biomechanics 42(3), 361-365 (2009)

17. Whittle, M.W.: Gait Analysis: An Introduction. Butterworth-Heinemann, Oxford (2007) 